Volume and Issues Obtainable at Center for Sustainability Research and Consultancy
Journal of Accounting and Finance in Emerging Economies
ISSN: 2519-0318\&ISSN (E):2518-8488
Volume 7: Issue 2June 2021
CSRᄃ
Journal homepage: www.publishing.globalcsrc.org/jafee

\title{
Forecasting Inflation Applying ARIMA Model with GARCH Innovation: The Case of Pakistan
}

Tahira Bano Qasim, Department of Statistics, The Women University Multan, Pakistan

*Hina Ali, Department of Economics, The Women University Multan, Pakistan

Natasha Malik, Department of Statistics, The Women University Multan, Pakistan

Malka Liaquat, Institute of Management Sciences, The Women University Multan, Pakistan

*Corresponding author's email address: hinaali@wum.edu.pk

\section{ARTICLE DETAILS \\ History \\ Revised format: May 2021 \\ Available Online: Jun 2021}

\section{Keywords}

Consumer Price Index,

Inflation, ARIMA, GARCH,

EGARCH, TGARCH

JEL Classification

E58, C43, C15

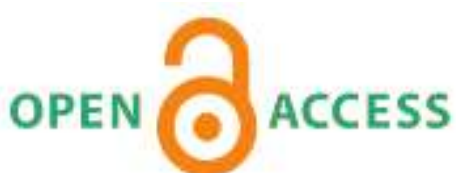

\section{ABSTRACT}

Purpose: The research aims to build a suitable model for the conditional mean and conditional variance for forecasting the rate of inflation in Pakistan by summarizing the properties of the series and characterizing its salient features.

Design/Methodology/Approach: For this purpose, Pakistan's Inflation Rate is based upon the Consumer Price Index (CPI), ranging from January 1962 to December 2019 has been analyzed. Augmented Dickey Fuller (ADF) test that was used for testing the stationarity of the series. The ARIMA modeling technique is a conditional mean and GARCH model for conditional variance. Models are selected on AIC and BIC model selection criteria. The estimating and forecasting ability of three ARIMA models with the GARCH $(2,2)$ model has been compared to capture the possible nonlinearity present in the data. To depict the possible asymmetric effect in the conditional variance, two asymmetric GARCH models, EGARCH and TGARCH models have been applied.

Findings: Based on statistical loss functions, GARCH $(2,2)$ model is the best variance model for this series. The empirical results reveal that the performance of model-2 is best for all the three variance models. However, the GARCH model is the best as the variance model for this series. This shows that the asymmetric effect invariance is not so important for the rate of inflation in Pakistan.

Implications/Originality/Value: The current study was based on the least considered variables and the pioneer in testing the complex relationship through the ARIMA model with GARCH innovation.

(C) 2021 The authors, under a Creative Commons AttributionNonCommercial 4.0

Recommended citation: Qasim, T. B., Ali, H., Malik, N. and Liaquat, M. (2021). Forecasting Inflation Applying ARIMA Model with GARCH Innovation: The Case of Pakistan. Journal of Accounting and Finance in Emerging Economies, 7 (2), 313-324 


\section{Introduction}

An increase in the extent of prices for products and services over a certain period resulting in a decrease in the purchasing capacity of a unit of currency is known as inflation. An increase in the money supply in a country is the major cause of inflation. Inflation Rate is the percent change in the overall price index, generally the Consumer Price Index (CPI), overtime is considered a good measure of inflation. The inflation rate is an important macroeconomic indicator and a key variable. Inflation forecasts provide a link between future inflation and current developments. For most central banks one of the most important tasks is to make monetary policy. While current inflation levels may provide scarce information for future planning, inflation forecasts are a key factor taken into account for making monetary policy. Inflation forecasts also play a critical role in the decision-making for stock valuations. Therefore, it is necessary to obtained reliable inflation forecasts.

The most important indicator in Pakistan to measure the price changes for everyday goods weighted appropriately to get precise measures is CPI. It also measures the direct or indirect effects of inflation on individuals and the household sector as a whole. It has been also employed by the Govt. sector as the official measure of inflation in formatting monetary policies. The CPI is also helpful in fixing wages and making policies about pension and retirement. Many business contracts, insurance premiums, rental agreements are routinely adjusted by taking account of changes in CPI.

Dynamics underlying Inflation can be captured using a univariate time series modeling approach. In a univariate time, series modeling approach, the behavior of a variable can be studied by exploring the nature of "dependence of current on its past." In the context of univariate time series modeling the most frequently applied methodology is autoregressive moving average (ARMA) models, and those models are subject to work in the hypothesis of stationarity but generally time series are non-stationary. In such situations, Nwankwo (2014) suggested an autoregressive integrated moving average (ARIMA) modeling approach in which nonstationary time sequence is transformed into stationary one by differencing up to appropriate order and then applying the ARMA modeling approach. These models also operate under the assumption of constant variance but the economic time series exhibit time-varying heteroscedasticity. To capture this phenomenon, the assumption of constant variance changes into non-constant. In recent years, Generalized Autoregressive Conditional Heteroscedasticity (GARCH) type models have become popular powerful tools for capturing the phenomenon of time-varying heteroscedasticity proposed by Engle (1982) and generalized by Bolerslev (1986). These models are successful in the case of symmetry in the variance. But sometimes there exist asymmetries in the variance due to different effects of good and bad news to the variance. To accommodate these asymmetries, some asymmetrical GARCH models for instance, "the Exponential GARCH (EGARCH) of Nelson (1991), GJR-GRCH model by Glosten, Jagannathan, and Runkle (1993), also known as Threshold GARCH (TGARCH), the Asymmetric Power ARCH (APARCH) by Ding, Granger, and Engle (1993) and the Quadratic GARCH (QARCH) by Santana et al. (1995), etc. are very popular in literature (Pasha, Qasim, and Aslam,2007).”

The primary objective of the current investigation is to model the conditional mean and variance and to forecast the rate of inflation in Pakistan by summarizing the properties of the series and characterizing its salient features. For this purpose, Pakistan's monthly inflation series based on Consumer Price Indices (CPI), ranging from January 1962 to December 2019 has been analyzed. A comparison has been carried out to assess the estimating and forecasting ability of ARIMA models as conditional mean with GARCH models for the conditional variance to capture the possible nonlinearity present in the data. To account for the possible asymmetries in the selected data, two asymmetric GARCH models, EGARCH and TGARCH models have been applied. The third stylized fact of the economic time series i.e., excess kurtosis has been accommodated by 
applying the quasi-maximum likelihood estimation technique which is robust to departure from normality. The comparison is carried out through statistical loss functions.

\section{Literature Review}

Ling and Li (1997) concentrated marginally integrated autoregressive moving-average in a time sequence model with conditional heteroscedasticity, which consolidated well-known generalized autoregressive conditional heteroscedastic (GARCH) and fractional (ARMA) models. Meyler, Kenny and Quinn (1998) judged two different approaches to the problem of classifying ARIMA models - the Box Jenkins approach and purpose penalty function methods. The focus was on the projected execution, which showed that the ARIMA estimate had been beaten.

Sekine (2001) assessed inflation function and anticipated one-year inflation for Japan, and found out that the marking connections, abundance cash, and yield gap were especially significant in the longer term a decisive factor in the balance model of inflation. Ratfai (2004) analyzed by placing store-level price data into bivariate Structural VAR models of inflation and relative cost asymmetry and assessed quantitative significance of distinctive pricing shocks in short-run aggregate price change dynamic.'Feridun (2006) used an autoregressive integrated moving average modeling technique to model and prediction inflation in Pakistan.

Mehmood (2012) applied Box and Jenkins methodology to forecast Pakistan's exports to SAARC, and found ARIMA $(1,1,4)$ as the best model, and concluded that Pakistan's exports to SAARC will increase in the future and recommended increasing the investment in that sector. Moffat and David (2016) used Box and Jenkins' methodology to mode the inflation rate in Nigeria and found that ARIMA $(0,0,1)$ is the best model to predict the rate of inflation in Nigeria. Makoni and Chikobvu (2018) applied the "seasonal autoregressive integrated moving average (SARIMA) model and generalized autoregressive conditional heteroscedasticity (GARCH)" model to inflation data in South Africa. SARIMA model of order $(1,1,0)(0,1,1)$ and GARCH model of order $(1,1)$ had been proved to be the best model for estimating and forecasting the inflation rate of South Africa. Sammy (2018) captured stochastic alteration of inflation rate data in Malaysia by applying GARCH models and exponential GARCH (EGARCH) models. Based on empirical results, it was concluded that the EGARCH model provided superior estimating ability as compared to the GARCH model. “

\section{Data and Methodology}

Generally, evaluation of time series is based upon the assumption that basic time-sequences are stationary but largely real-time series data is non-stationary. On the official level stationarity could be tested in the "autocorrelation function (ACF) and a partial autocorrelation function (PACF)" of causal series. Correlograms linked with ACF and PACF have also been believed the visual diagnostic instruments.

\section{ARIMA MODEL}

ARIMA models are the extension of the ARMA models to overcome the problem of nonstationary time series by introducing the integrated terms into the model. the resultant model denoted by ARIMA (p, d, q) has the form

$$
\emptyset(B) y_{t}=\theta(B) \varepsilon_{t}
$$

where $\emptyset(B)$, the generalized autoregressive of order $\mathrm{p}+\mathrm{d}$

i.e. $\emptyset(B)=\emptyset_{p}(B)(1-B)^{d}$

$$
=\emptyset_{p}(B) \nabla^{\mathrm{d}}
$$

Where $\emptyset_{p}(B)$ is a stationary autoregressive operator of order $\mathrm{p}$ and $\mathrm{d}$ is the differencing operator? 
1. For $\mathrm{AR}(p)$ the $\mathrm{ACF}$ decreases exponentially to zero while PACF is cutting after lag $p$.

2. For MA $(q)$ PACF falls exponentially to zero while ACF cuts off after lag $q$.

3. For one ARMA $(p, q)$ the ACF and PACF both decline exponentially to zero, and the identification of the orders $p$ and $q$ is complicated.

(for detail see Anderson (1977).

\section{Variance Models}

\section{Autoregressive Conditional Heteroscedastic (ARCH) Model}

To capture the dynamic behavior of variance, Engle (1982) suggested Auto-Regressive Conditional Heteroscedasticity process $(\mathrm{ARCH})$, which is the function of past error terms noted by $\operatorname{ARCH}(q)$ and is defined as

$$
\sigma_{t}^{2}=\alpha_{\circ}+\sum_{i=1}^{q} \alpha_{1} \varepsilon_{t-i}^{2}
$$

Where $\alpha_{\circ}>0, \alpha_{1} \geq 0$ for all $i=1,2, \ldots \ldots, q$

\section{Generalized Autoregressive Conditional Heteroscedastic (GARCH) Model}

In an empirical application of the $\operatorname{ARCH}(q)$ version, there is a relatively long lag in the conditional variance which creates the problem of negative variance and several parameter estimations. To solve this problem Bollerslev (1986) extended Engle's model by incorporating the past conditional variances in the ARCH model to avoid the negativity of the variance. The form of the $\operatorname{GARCH}(p, q)$ model is presented as

Where

$$
\sigma_{t}^{2}=\alpha_{0}+\sum_{i=1}^{q} \alpha_{t} \varepsilon_{t-i}^{2}+\sum_{j=1}^{\rho} \beta_{j} \sigma_{t-j}^{2}
$$

" $\alpha_{0}>0, \alpha_{i} \geq 0$ for all $i=1,2, \ldots, q$ and $\beta_{j} \geq 0$ all $j=0,1, \ldots, p$. "

\section{Exponential GARCH (EGARCH) Model}

Nelson (1991) was the first who characterized the asymmetries in the variance model due to the differential effect of positive and negative news, proposing the exponential GARCH (EGARCH). The model is given as "

$\ln \sigma_{t}^{2}=\alpha_{\circ}+\sum_{i=1}^{q}\left(\alpha_{i}\left|n_{t-i}-E\left(n_{t-i}\right)\right|++\gamma_{i} n_{t-i}\right)+\sum_{j-1}^{\rho} \beta_{j} \sigma_{t-j}^{2}$

Where $n_{t}=\frac{\varepsilon_{t}}{\sigma_{t}}$ is a standardized normal residual series"?

As the conditional variance is in logarithmic-linear form, therefore there is no need for positivity of the coefficients.

\section{Threshold GARCH (TGARCH) Model}

Threshold GARCH (TGARCH) also recognized as the GJR GARCH model is proposed by Gloston, Jagannathan, and Runkle (1993) by incorporating the asymmetric effect of the good and bad news in a quadratic form. The form of the $\operatorname{TGARCH}(p, q)$ model that

$$
\sigma_{t}^{2}=\alpha_{0}+\sum_{i=1}^{q}\left(\alpha_{i} \varepsilon_{t-i}^{2}+\gamma_{i} d_{t-i} \varepsilon_{t}^{2}-i\right)+\sum_{j=1}^{P} \beta j_{t j}^{\sigma^{2}}
$$

Where the dummy variable $d_{t}$ and using the value 0 positive shocks and 1 negative shock and

$$
\alpha_{\circ}>0, \alpha_{i} \geq 0 \gamma_{i} \geq 0
$$


for all $i=1,2, \ldots, q$ and $\beta_{j} \geq 0$ all $j=0,1, \ldots, p$.

\section{Forecast Evaluation Methods}

To compare, the forecasting performance of models considered in this study, some statistical measures of goodness such as Root Mean Square Error (RMSE), Mean Absolute Error (MAE), Mean Absolute Percentage Error, and Theil Inequality Coefficient (TIC) have been applied.

These are given as:

1. $R M S E=\sqrt{\frac{1}{h+1} \sum_{t-T}^{T+h}\left(r_{t+1}-\hat{r}_{t+1 T}\right)^{2}}$

2. $M A E=\frac{1}{h+1} \sum_{t=T}^{T+h}\left(\left|r_{t+1}-\hat{r}_{t+1 \mid T}\right|\right)$.

3. $M A P E=\frac{1}{h+1} \sum_{t=T}^{T+h}\left(\left|\frac{r_{t+1}-\hat{r}_{t+1 \mid T}}{r_{t+1}}\right|\right)$.

4. $T I C=\frac{\sqrt{\frac{1}{h+1} \sum_{t=T}^{T+h}\left(r_{t+1}-\hat{r}_{t+1 \mid T}\right)^{2}}}{\sqrt{\frac{1}{h+1} \sum_{t=T}^{T+h} \hat{r}_{t+1 \mid T}^{2}}+\sqrt{\frac{1}{h+1} \sum_{t=T}^{T+h}\left(r_{t+1}\right)^{2}}}$.

where $r_{t+1}$ is the observed value at time $t+1, \hat{r}_{t+1 \mid T}$ is the one step ahead forecasted value and $h$ is the number of forecasts.

A model using the lowest measure is considered the best model.

\section{Results and Discussion}

The data analyzed in this study is related to Pakistan's monthly inflation series based on Consumer Price Indices (CPI) ranging from January 1962 to December 2019 collected from web "www.tradingeconomics.com". The data contains 696 monthly observations via 684 observations are employed for estimation objectives and 12 observations for prediction evaluation.

Analysis based on the description of data, assessment of stationarity, developing the ARIMA of the series with the methods that were suggested by Box and Jenkins (1976), and developing of the conditional variance using GARCH- model. For this reason, the quantity of ARIMA models with increasing order has been evaluated to recognize the mean model. Basically, for the reason to identify the variance model, the number of GARCH models is assessed and the last model that has been selected relies on AIC and BIC. Such as time series should be subject to the asymmetries in the variance so two asymmetric GARCH models specifically EGARCH and TGARCH are considered for this investigation. These models alongside mean models are assessed and utilized for the prediction. For the choice of the last model for estimating the inflation series, some forecast evaluation methods have been utilized. E-Views3, E-Views7, Minitab, and Excel have been utilized as the main statistical software, for analysis. Fig 1 addresses the plot of the month-to-month inflation rate arrangement, uncovering the conduct of nonstationary series. Figure 4.1 represents the plot of the monthly inflation rate series, revealing the behavior of nonstationary series 


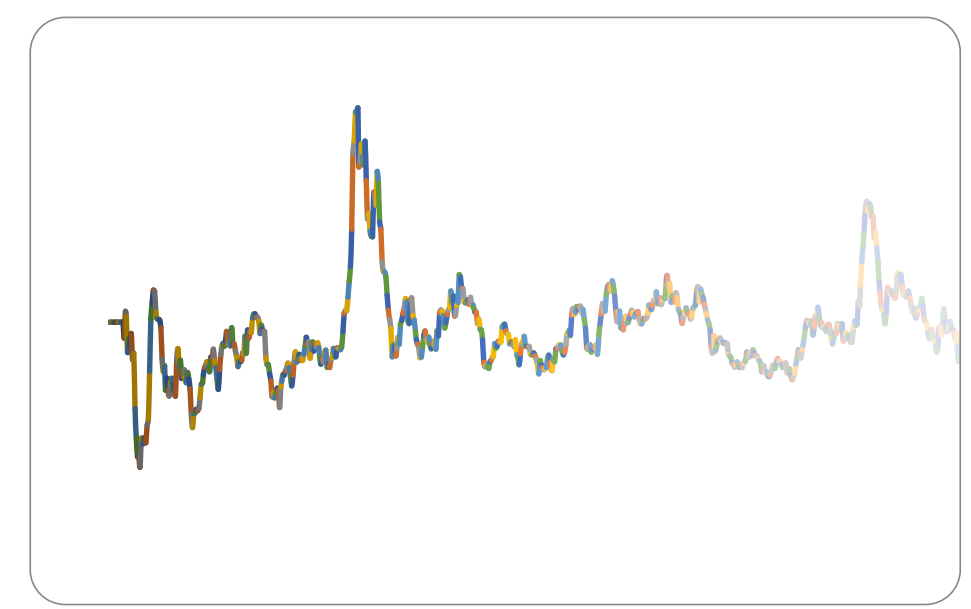

Figure 1: Plot of Monthly Inflation Rate

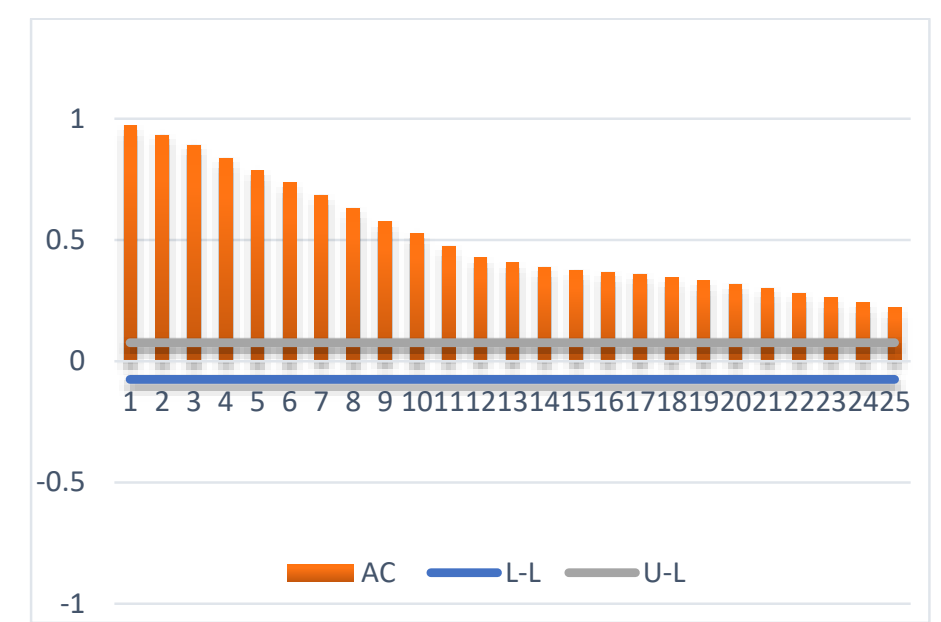

Fig 2: Correlogram of the Rate of Inflation

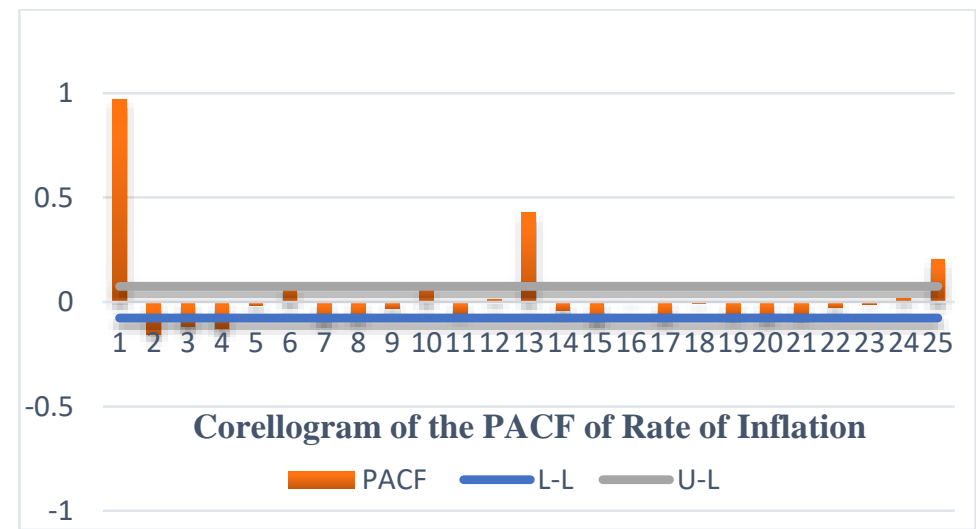

Fig 3: Corellogram of the PACF Rate of Inflation

To assess the stationarity of the Inflation Rate series, the Augmented Dickey-Fuller (ADF) unit root test has been applied and results are given in Table 1 .

Table 1: Augmented Dickey-Fuller (ADF)

\begin{tabular}{|c|c|c|}
\hline Data series & Test Statistic & Critical value \\
\hline Level & -2.292858 & $1 \% \quad-2.5687$ \\
\hline First difference & -11.27834 & $1 \% \quad-3.4423$ \\
\hline
\end{tabular}

Since the value of the test statistic is -2.292858 which leads to acceptance of null hypothesis of 
unit root at a $1 \%$ level of significance. However, the stationarity is achieved at first order as the null hypothesis of unit root is strongly denied at a $1 \%$ level of significance.

The Box and Jenkins (1976) methodology has been applied to model; the mean of the integrated series denoted by $r_{t}$. The first problem in ARMA modeling is the identification of the ARMA model. So, to identify the mean model, the number of ARMA models have been estimated to the given series with increasing order from ARMA $(0,0)$ to ARMA $(6,6)$, etc., and observe the correlogram of the residuals. Based on the inspection of ACF and PACF the model with the terms "AR (1), AR (2), AR (3), AR (4), AR (5), AR (6), MA (1), MA (12) is selected and named it as model 1."

\section{Model 1}

$" r_{t}=\emptyset_{0}+\emptyset_{1} r_{t-1}+\emptyset_{2} r_{t-2}+\emptyset_{3} r_{t-3}+\emptyset_{4} r_{t-4}+\emptyset_{5} r_{t-5}+\emptyset_{6} r_{t-6}+\theta_{1} \varepsilon_{t-1}+\theta_{12} \varepsilon_{t-12}+\varepsilon_{t} "$ The estimation results for this model are shown in the table

Table 2: Estimation Results for Model-1

\begin{tabular}{|l|l|l|l|l|}
\hline Variables & Co-efficient & Std. Error & Z-Statistic & Prob. \\
\hline C & 0.011180 & 0.015467 & 0.722840 & 0.4700 \\
\hline AR (1) & 0.181400 & 0.038648 & 4.693603 & 0.0000 \\
\hline AR (2) & 0.123072 & 0.038966 & 3.158423 & 0.0017 \\
\hline AR (3) & 0.053238 & 0.039154 & 1.359712 & 0.1744 \\
\hline AR (4) & 0.050457 & 0.038690 & 1.304119 & 0.1926 \\
\hline AR (5) & -0.080561 & 0.038390 & -2.098493 & 0.0362 \\
\hline AR (6) & 0.110925 & 0.037807 & 2.933933 & 0.0035 \\
\hline MA (1) & -0.079604 & 0.000199 & -400.8560 & 0.0000 \\
\hline MA (12) & -0.747518 & 0.025404 & -29.42476 & 0.0000 \\
\hline
\end{tabular}

Table 2 shows that the coefficients for AR (2), AR (6), MA (1), and MA (12) are highly significant and that for AR (3) and AR (4) are insignificant.

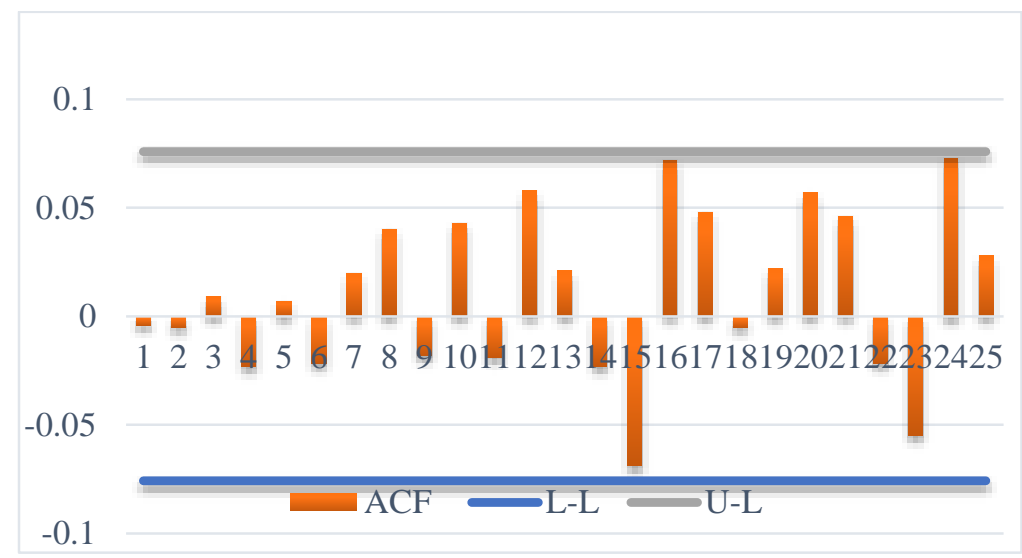

Fig: 4 Correlogram of the ACF of the residuals of Model-1 


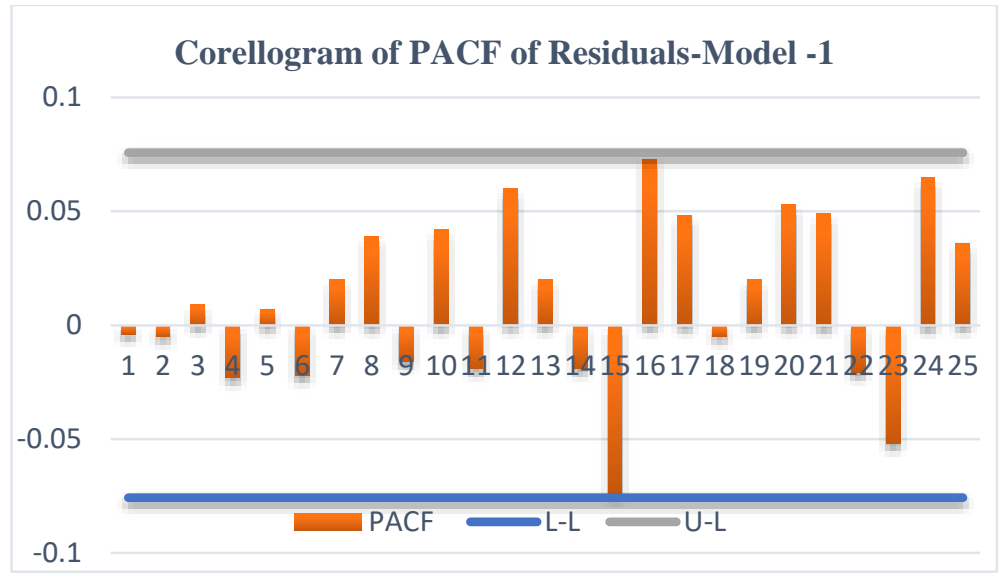

Fig: 5 Correlogram of PACF Residuals of Model-1

The terms AR (3) and AR (4) have been dropped and the model has been re-estimated and named Model 2. The specification of this model is

$$
r_{t}=\emptyset_{0}+\emptyset_{1} r_{t-1}+\emptyset_{2} r_{t-2}+\emptyset_{5} r_{t-5}+\emptyset_{6} r_{t-6}+\theta_{1} \varepsilon_{t-1}+\theta_{12} \varepsilon_{t-12}+\varepsilon_{t}
$$

Table: 3 Estimation Results for Model-2

\begin{tabular}{|lllll|}
\hline Variables & Coefficient & Std. Error & z-Statistic & Prob. \\
\hline C & 0.0111 & 0.0144 & 0.7732 & 0.4397 \\
\hline AR (1) & 0.1919 & 0.0382 & 5.0198 & 0.0000 \\
\hline AR (2) & 0.1388 & 0.0383 & 3.6272 & 0.0003 \\
\hline AR (5) & -0.0660 & 0.0378 & -1.7472 & 0.0811 \\
\hline AR (6) & 0.1218 & 0.0375 & 3.2523 & 0.0012 \\
\hline MA (1) & -0.0794 & 0.0002 & -402.2876 & 0.0000 \\
\hline MA (12) & -0.7445 & 0.0254 & -29.2657 & 0.0000 \\
\hline
\end{tabular}

Corellogram of PACF of the Residuals-Model-2

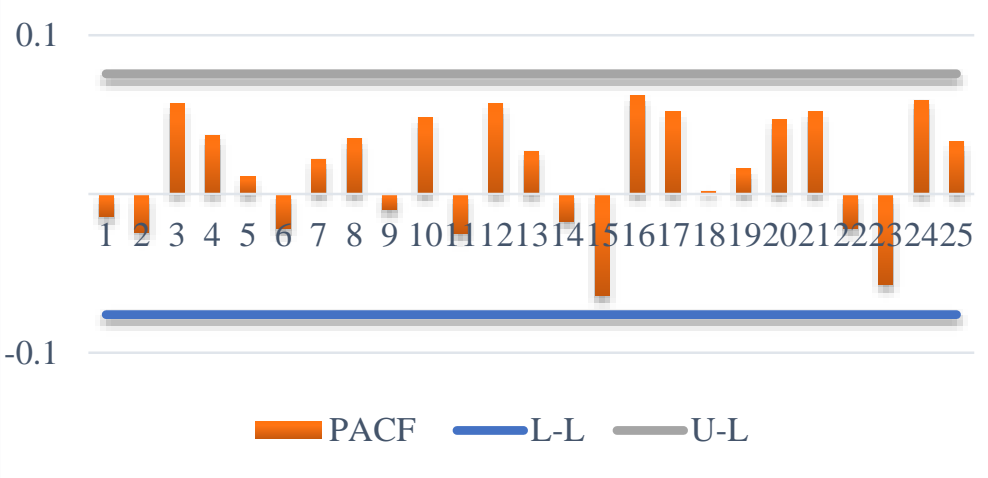

Fig: 6 Correlogram of PACF Residuals of Model-2 


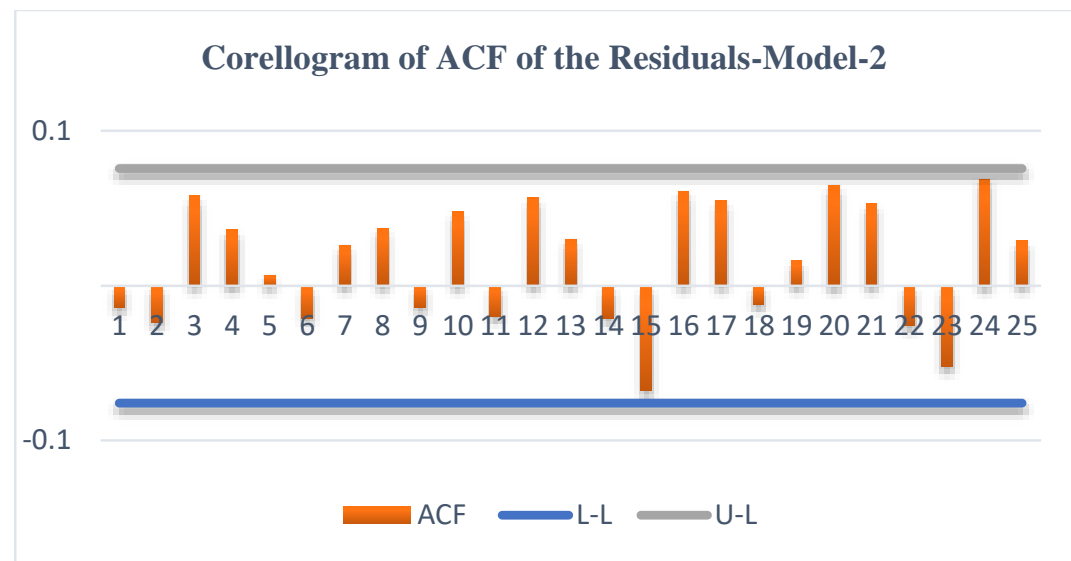

Fig: 7 Correlogram of ACF the Residuals Model-2

Corellogram of PACF of the Residuals-Model-2

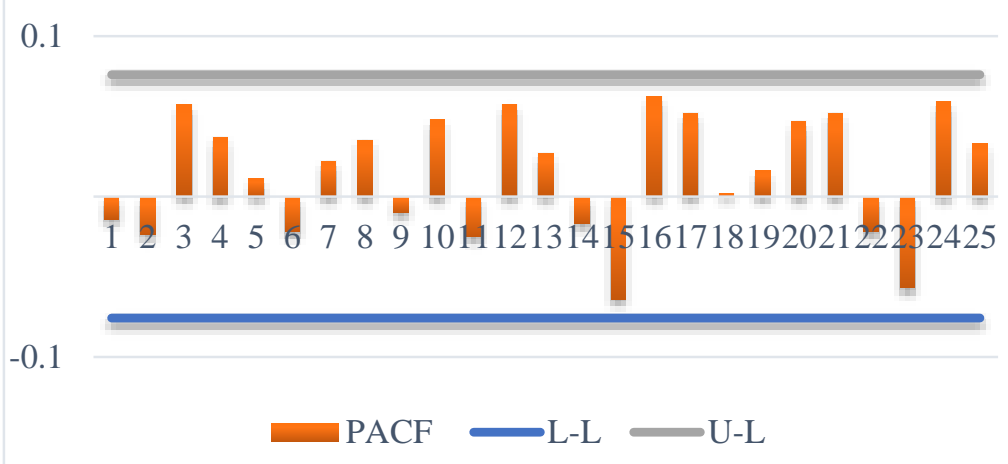

Fig: 8 Correlogram of PACF of the Residuals Model-2

The diagnosis is fulfilled for the new model, but the term AR (5) becomes insignificant at a 5\% level of significance. We also dropped this term and re-estimated the model. We call this new model Model-3. The specification of this model is

$$
r_{t}=\emptyset_{0}+\emptyset_{1} r_{t-1}+\emptyset_{2} r_{t-2}+\emptyset_{6} r_{t-6}+\theta_{1} \varepsilon_{t-1}+\theta_{12} \varepsilon_{t-12}+\varepsilon_{t}
$$

The estimation outcomes are given in Table 4.

Table 4: Estimation Results for Model-3

\begin{tabular}{|l|l|l|l|l|}
\hline Variable & Coefficient & Std. Error & t-Statistic & Prob. \\
\hline C & 0.011200 & 0.015202 & 0.736746 & 0.4615 \\
\hline AR (1) & 0.185613 & 0.038115 & 4.869749 & 0.0000 \\
\hline AR (2) & 0.133242 & 0.038205 & 3.487542 & 0.0005 \\
\hline AR (6) & 0.109226 & 0.036836 & 2.965229 & 0.0031 \\
\hline MA (1) & -0.079370 & 0.000195 & -406.7726 & 0.0000 \\
\hline MA (12) & -0.748127 & 0.025276 & -29.59873 & 0.0000 \\
\hline
\end{tabular}

"Table 4 shows that coefficients for AR (1), AR (2), AR (6), MA (1), and MA (12) in this model are highly significant, and constant-coefficient is insignificant." 


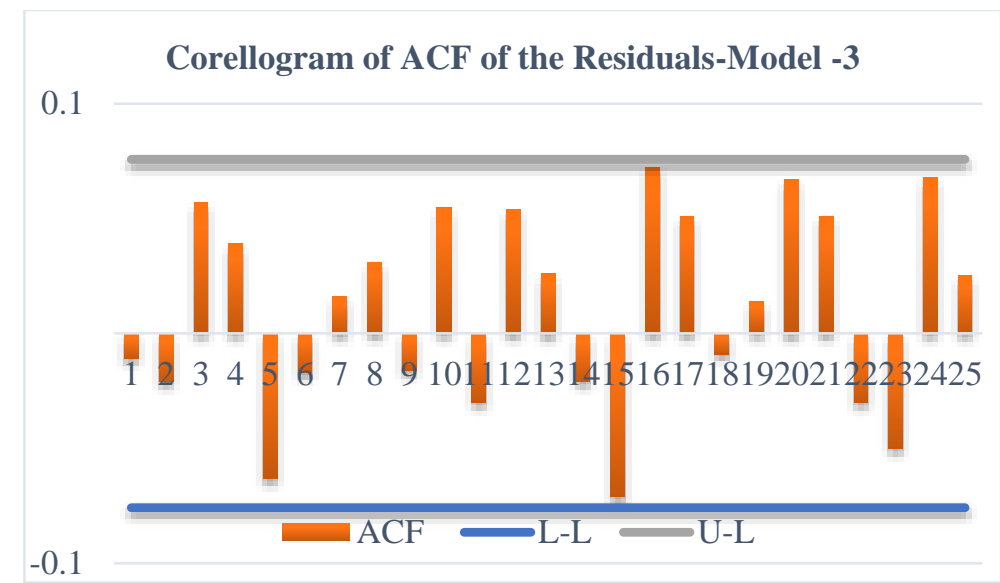

Fig: 9 Correlogram of ACF of the Residuals Model-3

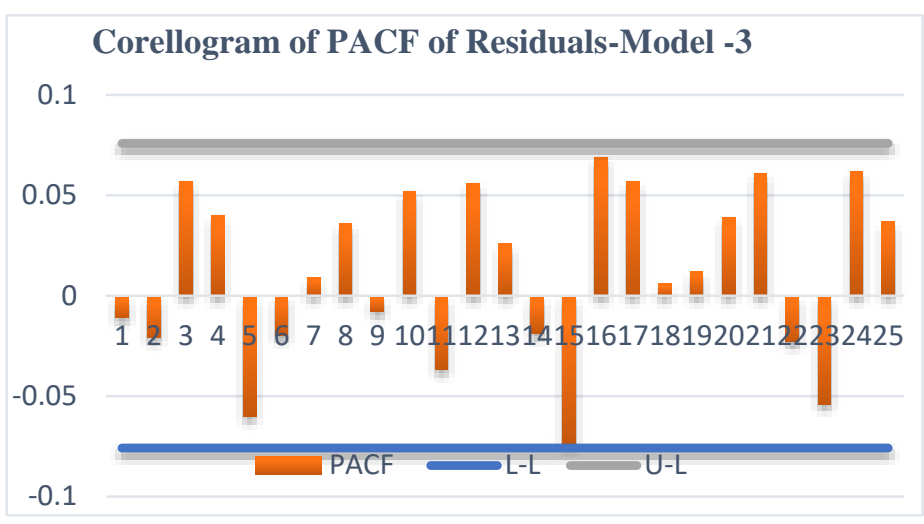

Fig: 10 Correlogram of PACF of the Residuals Model-3

During the estimation of mean models in diagnostic checking, we have observed that the ACF and PACF of the squared residual values are significant (the results are not reported here) showing that the residuals are conditionally heteroscedastic. To model this conditional heteroscedasticity, GARCH models initially proposed by Engle (1982) and generalized by Bollerslev (1986) are considered the best models in the literature. So, we have applied these models to overcome this difficulty. To identify the order of GARCH models we have estimated Model-1, Model-2, and model-3 with increasing order of GARCH models. The strategy employed when selecting the right model in the competitive models is built on the Akaike information criterion (AIC) by Akaike (1971), Bayesian information criterion (BIC) by Schwarz (1978), and maximum likelihood criterion. The results for some models are given in the following Tables and these results reveal that GARCH $(2,2)$ is appropriate with Model-1, 2, and 3.

Table 5: Selection of GARCH Model with Model-1

\begin{tabular}{|l|l|l|l|}
\hline Model specification & AIC & BIC & Log-likelihood \\
\hline GARCH $(1,1)$ & 2.945667 & $\mathbf{3 . 0 2 5 7 4 4}$ & -985.1084 \\
\hline GARCH $(1,2)$ & 2.946228 & 3.032978 & -984.2982 \\
\hline GARCH $(2,2)$ & $\mathbf{2 . 9 4 5 1 7 6}$ & 3.038599 & $\mathbf{- 9 8 2 . 9 4 2 0}$ \\
\hline
\end{tabular}

Table 6: Selection of GARCH Model with Model-2

\begin{tabular}{|l|l|l|l|}
\hline Model specification & AIC & BIC & Log-likelihood \\
\hline GARCH $(1,1)$ & 2.9447 & $\mathbf{3 . 0 1 1 5}$ & -986.8039 \\
\hline GARCH $(1,2)$ & 2.9450 & 3.0184 & -985.8961 \\
\hline GARCH $(2,2)$ & $\mathbf{2 . 9 4 4 1}$ & 3.0242 & $\mathbf{- 9 8 4 . 6 0 3 4}$ \\
\hline
\end{tabular}


Table 7: Selection of GARCH Model with Model-3

\begin{tabular}{|l|l|l|l|}
\hline Model specification & AIC & BIC & Log-likelihood \\
\hline GARCH $(1,1)$ & 2.943925 & $\mathbf{3 . 0 0 3 9 8 3}$ & -987.5186 \\
\hline GARCH $(1,2)$ & 2.943838 & 3.010569 & -986.4892 \\
\hline GARCH $(2,2)$ & $\mathbf{2 . 9 4 2 0 2 0}$ & 3.015424 & $\mathbf{- 9 8 4 . 8 7 3 7}$ \\
\hline
\end{tabular}

\section{Forecast evaluation}

We have obtained the forecasts using these models and also by replacing identified GARCH models with EGARCH and TGARCH models, from January to December of the year 2019. The forecast assessment based on Statistical loss functions the mean absolute error (MAE); the root means square error (RMSE); and Thieves $\mathrm{U}$ and the mean absolute percent error (MAPE).

Table 8: Forecast Evaluation

\begin{tabular}{|l|l|l|l|l|}
\hline Models & RMSE & MAE & MAPE & TIC \\
\hline Model-1-GARCH (2,2) & 0.7568 & 0.6057 & 56.5474 & 0.4804 \\
\hline Model-2-GARCH (2,2) & 0.7418 & 0.5898 & 55.0522 & 0.4724 \\
\hline Model-3-GARCH (2,2) & 0.7517 & 0.5997 & 56.1148 & 0.4790 \\
\hline Model-1-EGARCH (2,2) & 0.8254 & 0.6479 & 62.0887 & 0.5099 \\
\hline Model-2-EGARCH (2,2) & 0.8008 & 0.6264 & 59.4865 & 0.4994 \\
\hline Model-3-EGARCH (2,2) & 0.7937 & 0.6274 & 56.7304 & 0.5017 \\
\hline Model-1-TGARCH (2,2) & 0.8194 & 0.6441 & 59.8485 & 0.5078 \\
\hline Model-2-TGARCH (2,2) & 0.7988 & 0.6249 & 58.2355 & 0.4975 \\
\hline Model-3-TGARCH (2,2) & 0.8072 & 0.6346 & 58.3471 & 0.5042 \\
\hline
\end{tabular}

The above result shows that Model-2-GARCH $(2,2)$ is the best to forecast the series. So, we may is the best model among competing models to predict the rate of inflation in Pakistan.

\section{Summary and Conclusion}

The key objective of this study is to highlight the statistical features present in the Inflation data in Pakistan and to model and forecast Pakistan's Inflation Rate. Into the context of mean modeling, Box and Jenkins methodology is used to model the rate of inflation. For this reason, the number of ARIMA models has been calculated to identify the mean model. The statistical tools used to identify the mean model are ACF, PACF of the residuals of the estimated models. $\mathrm{ACF}$ and PACF of the squared residual values of the mean models have been used to identify the order of the GARCH models as the variance model.

So, in our study, the number of GARCH models is estimated with increasing order of GARCH. The last model was chosen based on the AIC and BIC. Order in which the GARCH models for all the models, mentioned above is $(2,2)$. To accommodate the asymmetric effect in the variance model two asymmetric GARCH models namely EGARCH and TGARCH are also applied along with each of the three selected mean models. Those models are anticipated and applied for predictions.

For a choice of the last model for predicting the inflation series, the prediction of the competing models is evaluated by using the statistical loss functions. The empirical results reveal that the performance of model-2 is best for all the three variance models. However, the GARCH model is the best as the variance model for this series. This shows that the asymmetric effect invariance is 
not so important for the rate of inflation in Pakistan.

\section{References}

Bollerslev, T. (1986). Generalized autoregressive conditional heteroskedasticity. Journal of econometrics, 31(3), 307-327.

Ding, Z., Granger, C. W., \& Engle, R. F. (1993). A long memory property of stock market returns and a new model. Journal of empirical finance, 1(1), 83-106.

Engle, R. F. (1982). Autoregressive conditional heteroscedasticity with estimates of the variance of United Kingdom inflation. Econometrica: Journal of the econometric society, 9871007.

Feridun, M. (2006). Forecasting Inflation in Developing Nations: The Case of Pakistan. 138-159.

Glosten, L. R., Jagannathan, R., \& Runkle, D. E. (1993). On the relation between the expected value and the volatility of the nominal excess return on stocks. The journal of finance, 48(5), 1779-1801.

Ling, S., \& Li, W. K. (1997). On fractionally integrated autoregressive moving-average time series models with conditional heteroscedasticity. Journal of the American Statistical Association, 92(439), 1184-1194.

Makoni, T., \& Chikobvu, D. (2018). Modeling tourism demand volatility using a seasonal autoregressive integrated moving average autoregressive conditional heteroscedasticity model for Victoria Falls Rainforest arrivals in Zimbabwe. Journal of Economic and Financial Sciences, 11(1), 1-9.

Mehmood. S (2012): Forecasting Pakistan's Exports to SAARC: An Application of Univariate ARIMA Model. Journal of Contemporary Issues in Business Research Vol 1, Issue No.3, 96-110.

Meyler, A., Kenny, G., \& Quinn, T. (1998). Forecasting Irish inflation using ARIMA models. 148.

Moffat I. U. and David A. E. (2016): MODELING INFLATION RATES IN NIGERIA: BOXJENKINS' APPROACH. International Journal of Mathematics and Statistics Studies Vol.4, No.2, 20-27.

Nelson, D. B. (1991). Conditional heteroskedasticity in asset returns: A new approach. Econometrica: Journal of the Econometric Society, 347-370.

Nwankwo, S. C. (2014). Autoregressive integrated moving average (ARIMA) model for the exchange rate (Naira to Dollar). Academic Journal of Interdisciplinary Studies, 3(4), 429.

Pasha, G. R., Qasim, T., \& Aslam, M. (2007). Estimating and forecasting volatility of financial time series in Pakistan with GARCH-type models.

Ratfai, A. (2004). Inflation and relative price asymmetry. Available at SSRN 296244.

SAMMY, O. F. (2018). MODELLING RATES OF INFLATION IN KENYA: AN APPLICATION OF GARCH AND EGARCH MODELS (Doctoral dissertation, JKUAT-COPAS).

Santana, A., Saxena, B., Noble, N. A., Gold, L. I., \& Marshall, B. C. (1995). Increased expression of transforming growth factor-beta isoforms (beta 1, beta 2, beta 3 ) in bleomycin-induced pulmonary fibrosis. American journal of respiratory cell and molecular biology, 13(1), 34-44.

Sekine, T. (2001). Modeling and forecasting inflation in Japan. 